Revista Brasileira de

Engenharia Agrícola e Ambiental

v.17, n.1, p.47-53, 2013

Campina Grande, PB, UAEA/UFCG - http://www.agriambi.com.br

Protocolo 089.11 - 06/05/2011 • Aprovado em 26/10/2012

\title{
Resíduos de serragem de mármores como corretivo da acidez de solo
}

\author{
Valério Raymundo ${ }^{1}$, Mirna A. Neves ${ }^{2}$, M arcos S. N. Cardoso ${ }^{3}$, \\ Izaias S. Bregonci ${ }^{4}$, Julião S. S. Lima ${ }^{5} \&$ Arthur B. Fonseca ${ }^{2}$
}

\begin{abstract}
RESU MO
O grande volume de resíduos gerados pela indústria de rochas ornamentais e sua composição com elementos essenciais à produção vegetal, indicam possibilidades de aproveitamento como corretivo de acidez de solos. Este estudo teve como objetivo a comparação em relação à capacidade de neutralização de acidez de solo, entre um resíduo de mármore serrado em tear diamantado, outro serrado em tear convencional e um calcário comercial. No experimento utilizaram-se, como substrato, amostras de solo classificado como Latossolo Vermelho-Amarelo distrófico e três materiais testados em quatro diferentes níveis $(75,100,150,300 \%$ e testemunha) de uma recomendação padrão de correção de solo. As unidades experimentais foram acondicionadas em sacos de polietileno elevando-se a umidade do solo a $70 \%$ da capacidade de campo com água destilada. 0 delineamento experimental foi inteiramente casualizado, em esquema fatorial de $3 \times 4+1$ (testemunha) com 4 repetições totalizando 52 unidades experimentais. Foram analisados o pH em água, $\mathrm{Ca}, \mathrm{Mg}, \mathrm{Al}, \mathrm{H}+\mathrm{Al}$ e calculados SB, CTC, V e m. Os resíduos apresentaram bom potencial para utilização como corretivo de acidez de solo, com reatividade inicial superior à do calcário comercial.
\end{abstract}

Palavras-chave: rochas ornamentais, calagem, agrominerais

\section{Marble cutting wastes as amendment of soil acidity}

\section{ABSTRACT}

The great volume of calcareous wastes generated by dimension stone industry and its composition, rich in important elements to promote plant growth, indicate possibilities of utilizing them as amendment of soil acidity. This study aimed to compare the capacity of neutralization of soil acidity and the use of two kinds of marble waste and a commercial limestone. The experiment was conducted in a completely randomized design, in factorial scheme, with four replications. An Oxisol mixed with four different doses of amendment $(75,100,150,300 \%$ and control), based on a standard recommendation. The experimental units consited of storage in polyethylene bags, and the soil moisture was elevated up to $70 \%$ of field capacity using distillated water. The $\mathrm{pH}, \mathrm{Ca}, \mathrm{Mg}, \mathrm{Al}, \mathrm{H}+\mathrm{Al}$, were analysed and SB, CTC, V were calculated. The wastes showed good potential to be used as amendment of soil acidity, since the initial reactivity was higher than the commercial limestone.

Key words: dimension stones, liming, agrominerals

\footnotetext{
SIN DIROCHAS, Rua Vinte e Cinco de Março 1/3, Edifício Jorge Miguel 60 andar, Centro, CEP 29300-100, Cachoeiro de Itapemirim, ES. Fone: (28) 9935-0417. E-mail: valerio30@gmail.com

2 Departamento de Geologia/U FES, Alto Universitário s.n., CEP 29500-000, Alegre, ES. Fone: (28) 3552-8698. E-mails: mirna.neves@ufes.br: arthurbazoni@yahoo.com.br

${ }^{3}$ IN CAPER, Av. Dr. Olívio Correa Pedrosa, 566, CEP 29500-000, Alegre, ES. Fone: (28) 3552-4125. E-mail: izaias@incaper.es.gov.br

${ }^{4}$ SAAE/Alegre, Av. Dr. Olívio Correa Pedrosa, s.n., CEP 29500-000, Alegre, ES. Fone: (28) 8808-2169. E-mail: marcos.snc@gmail.com

${ }^{5}$ D epartamento de Engenharia Rural/U FES, Alto U niversitário s.n., CEP 29500-000, Alegre, ES. Fone: (28) 3552-8628. E-mail: juliao.lima@ufes.br
} 


\section{INTRODUÇÃO}

O estado do Espírito Santo lidera a produção brasileira de rochas ornamentais (mármores e granitos para revestimento) colocando o setor como um dos pilares da economia capixaba e importante fonte de empregos (Calmon \& Silva, 2006). A relevância desta indústria para o desenvolvimento regional é inquestionável mas a extração e o beneficiamento de rochas podem causar danos ao meio ambiente, pela falta de gestão de resíduos.

Durante o processo de beneficiamento os blocos de rocha extraídos da pedreira são serrados em chapas as quais, posteriormente, são polidas e cortadas para a fabricação de ladrilhos e outros produtos (Moreira et al., 2003). A serragem de blocos é feita em dois tipos de teares: um deles usa lâminas de aço e o outro usa lâminas ou fios diamantados (Calmon \& Silva, 2006).

O processo em que se usam lâminas de aço, também conhecido como sistema convencional, ainda é o mais utilizado no Brasil (Almeida \& Chaves, 2002). Neste processo os teares são dotados de lâminas com movimento pendular por onde circula uma polpa de serragem, também conhecida como "lama abrasiva", composta por granalha de aço (grãos milimétricos facetados), cal e água (Braga et al., 2010). Esta mistura é bombeada homogeneamente sobre os blocos, logo acima das lâminas. O atrito entre lâmina, granalha e rocha, causa cominuição ou diminuição do tamanho da granalha, que perde seu poder abrasivo. A lama é então descartada compondo um resíduo constituído por pó de rocha, cal e finos metálicos provenientes do desgaste das lâminas e da granalha (Braga et al., 2010).

No sistema de serragem com lâminas diamantadas não é necessário o uso de granalha e cal, utilizando-se apenas água no processo; assim, o resíduo gerado é composto, basicamente, de pó de rocha e água (Moreira et al., 2003).

O uso desses materiais como matéria-prima em outros processos produtivos pode transformar resíduos em subprodutos úteis diminuindo as grandes quantidades que são depositadas em aterros e contribuindo com a minimização dos impactos ambientais (Manhães \& Holanda, 2008).

Trabalhos realizados relatam a possibilidade de utilização de pó de rocha no enriquecimento mineral e na correção de acidez de solos, processo conhecido como rochagem (Fyfe et al., 2006; Theodoro \& Leonardos, 2006). A rochagem tem mostrado efeitos positivos sobre a qualidade química de solos degradados (Silva et al., 2008) e representam uma alternativa de baixo custo e ambientalmente segura para agricultores dos países em desenvolvimento (Straaten, 2006).

É importante destacar que a demanda por corretivos agrícolas no sul do estado do Espírito Santo é grande, colocando a calagem como uma boa alternativa para utilização dos resíduos de serragem de mármores (rochas carbonáticas ricas em $\mathrm{Ca}$ e/ou $\mathrm{Mg}$ ). A calagem propicia o aumento do $\mathrm{pH}$, fornecimento de $\mathrm{Ca}$ e $\mathrm{Mg}$ para o solo, neutralização do $\mathrm{Al}$ trocável, modificações da capacidade de troca catiônica efetiva e alteração da disponibilidade de micronutrientes podendo, inclusive, elevar os teores foliares de $\mathrm{Ca}$ e $\mathrm{Mg}$ (Natale et al., 2007). Abreu Júnior et al. (2003) encontraram, estudando solos brasileiros com $\mathrm{pH}$ inferior a 5,6, correlações positivas entre o
pH e os valores de P, Ca, Mg, K, SB, CTC e V\%, e negativas com a saturação de $\mathrm{Al}$, que foi o cátion trocável predominante em $32 \%$ dos solos estudados.

De acordo com Ramos et al. (2006) o calcário é o corretivo mais utilizado porém tem lenta mobilidade no perfil do solo, sendo lenta a correção, além da camada de incorporação. Neste sentido o uso de resíduos provenientes da serragem de mármore poderia ter seus efeitos acelerados devido à fina granulação desses materiais.

Propôs-se, com este trabalho, investigar a possibilidade de utilização de resíduos de serragem de mármores da região de Cachoeiro de Itapemirim, ES, como corretivo de acidez de solo.

\section{Material e MÉTODOS}

O experimento foi realizado no Centro de Ciências Agrárias da Universidade Federal do Espírito Santo (CCA/UFES) localizado na cidade de Alegre, sul do estado do Espírito Santo, entre os meses de maio e agosto de 2008.

O delineamento experimental foi inteiramente casualizado, em esquema fatorial de $3 \times 4+1$ (testemunha) com 4 repetições totalizando 52 unidades experimentais. O cálculo da dosagem para correção de acidez foi feito com base no PRNT dos materiais utilizados como corretivo e nos dados da análise do solo (CFSEMG, 1999). A necessidade de calcário foi calculada em $1.490 \mathrm{~kg}$ por hectare e, a partir deste valor, foram definidas doses de 75, 100, 150 e $300 \%$ da dose recomendada (Tabela 1) além da testemunha, sem dosagem de corretivo. Os resíduos utilizados foram gerados na serragem dos mármores mais comumente processados pelas indústrias de Cachoeiro de Itapemirim, ES: um serrado em tear com lâmina diamantada(LD) e outro serrado em tear com lâmina e granalha de aço (LA). Para fins de comparação do poder de neutralização de acidez do solo utilizou-se também um calcário comercial (CC).

Tabela 1. Doses de resíduos (LD e LA) e de calcário comercial (CC) recomendadas com base no PRNT e nas características do solo, definindo os diferentes tratamentos do experimento $(\mathrm{Q} C=$ quantidade de calcário $)$

\begin{tabular}{|c|c|c|c|c|c|c|}
\hline & \multirow{2}{*}{ PRNT } & \multirow{2}{*}{$\begin{array}{c}\mathrm{QC} \\
\left(\mathrm{t} \mathrm{h} \mathrm{a}^{-1}\right)\end{array}$} & \multicolumn{4}{|c|}{ Doses (t ha-1 ${ }^{-1}$} \\
\hline & & & $75 \%$ & $100 \%$ & $150 \%$ & $300 \%$ \\
\hline LD & 102,72 & 1,45 & 1,09 & 1,45 & 2,17 & 4,34 \\
\hline LA & 99,55 & 1,49 & 1,12 & 1,45 & 2,24 & 4,48 \\
\hline CC & 86,11 & 1,73 & 1,29 & 1,73 & 2,59 & 5,18 \\
\hline
\end{tabular}

LD - resíduo do tear de lâmina diamantada, LA - resíduo do tear de lâmina de aço, CC - calcário comercial

Os ensaios de peneiramento e a medição dos teores de $\mathrm{Ca}$ e Mg para determinação do Poder Relativo de Neutralização Total (PRNT) foram feitos de acordo com a metodologia oficial (Brasil, 1983). Os valores obtidos para o PRNT dos resíduos LA e LD (Tabela 2) mostram que eles possuem granulação de calcário "filler" mais fina que $0,3 \mathrm{~mm}$ (peneira ABNT50) com reatividade igual ou superior a $100 \%$, ultrapassando o valor obtido para o CC.

O solo utilizado (Latossolo Vermelho-Amarelo distrófico) é representativo da natureza ácida e topografia acidentada ocupada 
Tabela 2. Caracterização dos resíduos e do calcário comercial usados no experimento como corretivo de acidez de solo

\begin{tabular}{|c|c|c|c|c|c|c|c|}
\hline \multirow{2}{*}{$\begin{array}{l}\text { Material } \\
\text { testado* }\end{array}$} & PRNT & PN & $\mathrm{CaO}$ & $\mathrm{MgO}$ & \multicolumn{3}{|c|}{ Peneira ABNT (mm) } \\
\hline & \multicolumn{4}{|c|}{$(\%)$} & 2,0 & 0,84 & 0,3 \\
\hline 10 & 102,7 & & 343 & & 6 & & \\
\hline LA & & & & & & & \\
\hline $\mathrm{CC}$ & 86,11 & 92,00 & 32,00 & 14,00 & $100 \%$ & $89,00 \%$ & $85,00 \%$ \\
\hline
\end{tabular}

* LD - resíduo do tear de lâmina diamantada, LA - resíduo do tear de lâmina de aço, CC - calcário comercial

por pastagens da região de Alegre, sul do estado do Espírito Santo. A coleta de solo foi feita com utensílio de aço em profundidade de $20 \mathrm{~cm}$ em um percurso "ziguezague"; o material foi secado ao ar, peneirado em malha de $2 \mathrm{~mm}$ e armazenado em sacos plásticos; após o quarteamento foram recolhidas amostras para caracterização química inicial (Tabela 3).

As análises seguiram a metodologia proposta pela EMBRAPA (1997). Foram determinados os seguintes atributos químicos do solo: $\mathrm{pH}$; teores de cálcio $(\mathrm{Ca})$, magnésio $(\mathrm{Mg})$ e alumínio (Al); acidez potencial $(\mathrm{H}+\mathrm{Al})$; saturação de alumínio (m); soma de bases (SB); saturação em bases (V) e capacidade de troca catiônica (CTC). A interpretação dos valores para os atributos avaliados foi feita de acordo com os preceitos de Fullin \& Dadalto (2001).

Os materiais testados como corretivo foram pesados e incorporados às amostras de $2 \mathrm{dm}^{3} \mathrm{de}$ solo. As amostras foram armazenadas em sacos de polietileno e umedecidas com água destilada a $70 \%$ da capacidade de campo. Após 30 dias de incubação foram analisados os mesmos parâmetros definidos na análise inicial do solo.

A análise estatística dos dados foi feita com o programa SAEG 9.1, em que os dados foram submetidos à análise de variância pelo teste $\mathrm{F}$; os efeitos dos tratamentos estudados foram: tipo de material utilizado como corretivo, doses aplicadas e as interações entre ambos. Realizaram-se análises de regressão para os dados quantitativos e o teste de Tukey a $5 \%$ de probabilidade foi aplicado nos dados qualitativos. Os dados quantitativos que não tiveram ajuste pela análise de regressão também foram analisados pelo teste de Tukey.

A interpretação dos resultados em relação aos níveis dos atributos, foi feita com base nas determinações da EMBRAPA (2010) para o cultivo do milho.

\section{RESULTADOS E DISCUSSÃO}

Os atributos químicos do solo $(\mathrm{pH}, \mathrm{Mg}, \mathrm{Al}, \mathrm{H}+\mathrm{Al}, \mathrm{m}, \mathrm{V}$ e CTC) responderam significativamente à aplicação do corretivo evidenciando interação pela análise de variância; a SB apresentou significância isoladamente em relação aos materiais testados como corretivo e às doses aplicadas enquanto o $\mathrm{Ca}$ evidenciou significância apenas para a dose.

\section{Influência dos materiais testados como corretivo}

Na dosagem de $100 \%$, recomendada pelo método adotado neste trabalho, todas as fontes de corretivo utilizadas (LA, LD e CC) foram suficientes para neutralizar a acidez trocável (Al) e corrigir o $\mathrm{pH}$ para a faixa mínima ideal para o desenvolvimento da maioria das culturas agrícolas, que varia de 5,5 a 6,5, conforme constatado na literatura nacional e estando de acordo com resultados encontrados por Flora et al. (2007); no entanto, o fornecimento de $\mathrm{Ca}$ e $\mathrm{Mg}$ e a elevação da saturação de bases (V) só ocorreram de forma satisfatória na dosagem de 300\%, ou seja, três vezes acima da dosagem recomendada (Tabela 4) o que se deve ao fato de o método de recomendação de calagem utilizado ser suficiente para neutralização do Al tóxico às plantas e para elevar os teores de Ca e $\mathrm{Mg}$ até valores próximos de 2,0 cmol $\mathrm{dm}^{-3}$, e não mais.

A acidez potencial $(\mathrm{H}+\mathrm{Al})$ foi gradativamente reduzida com o aumento da dosagem de corretivo para todas as fontes utilizadas, havendo diferença significativa entre as fontes

Tabela 4. Valores médios dos parâmetros analisados no solo tratado com resíduo do tear com lâmina de aço (LA), resíduo do tear diamantado (LD) e calcário comercial (CC)

\begin{tabular}{|c|c|c|c|c|}
\hline \multirow{2}{*}{ Parâmetro } & \multicolumn{4}{|c|}{ Dose $(\%)$} \\
\hline & 75 & 100 & 150 & 300 \\
\hline \multicolumn{5}{|c|}{$\mathrm{pH}$} \\
\hline $\mathrm{LA}$ & $5,4 a$ & $5,5 a$ & $6,0 \mathrm{a}$ & $6,6 b$ \\
\hline LD & $5,4 a b$ & $5,6 a$ & $6,0 a b$ & $6,9 a$ \\
\hline $\mathrm{CC}$ & $5,3 \mathrm{~b}$ & $5,5 \mathrm{a}$ & $5,9 \mathrm{~b}$ & $6,5 \mathrm{c}$ \\
\hline \multicolumn{5}{|c|}{$\mathrm{Mg}\left(\mathrm{cmol}_{\mathrm{c}} \mathrm{dm}^{-3}\right)$} \\
\hline LA & $0,5 \mathrm{a}$ & $0,5 a b$ & $0,7 \mathrm{a}$ & $0,9 \mathrm{~b}$ \\
\hline LD & $0,5 \mathrm{a}$ & $0,6 a$ & $0,8 \mathrm{a}$ & $1,1 \mathrm{a}$ \\
\hline $\mathrm{CC}$ & $0,4 a$ & $0,4 b$ & $0,5 b$ & $0,7 \mathrm{C}$ \\
\hline \multicolumn{5}{|c|}{$\mathrm{Al}\left(\mathrm{cmol}_{\mathrm{c}} \mathrm{dm}^{-3}\right)$} \\
\hline LA & $0,1 \mathrm{a}$ & $0,1 \mathrm{a}$ & $0,0 \mathrm{a}$ & $0,0 \mathrm{a}$ \\
\hline LD & $0,1 \mathrm{a}$ & $0,0 a b$ & $0,0 \mathrm{a}$ & $0,0 \mathrm{a}$ \\
\hline $\mathrm{CC}$ & $0,0 \mathrm{~b}$ & $0,0 \mathrm{~b}$ & $0,0 \mathrm{a}$ & $0,0 \mathrm{a}$ \\
\hline \multicolumn{5}{|c|}{$\mathrm{H}+\mathrm{Al}\left(\mathrm{cmol}_{\mathrm{c}} \mathrm{dm}^{-3}\right)$} \\
\hline LA & $3,3 \mathrm{a}$ & $3,0 \mathrm{a}$ & $2,5 \mathrm{a}$ & $1,8 \mathrm{a}$ \\
\hline LD & $3,4 a$ & $3,0 \mathrm{a}$ & $2,5 a$ & $1,1 b$ \\
\hline $\mathrm{CC}$ & $3,3 \mathrm{a}$ & $3,1 \mathrm{a}$ & $2,3 a$ & $1,7 \mathrm{a}$ \\
\hline \multicolumn{5}{|c|}{$\mathrm{m}(\%)$} \\
\hline LA & $8,2 \mathrm{a}$ & $3,5 \mathrm{a}$ & $0,6 a$ & $0,0 \mathrm{a}$ \\
\hline LD & $9,7 a$ & $0,9 a b$ & $0,0 \mathrm{a}$ & $0,0 \mathrm{a}$ \\
\hline $\mathrm{CC}$ & $0,0 \mathrm{~b}$ & $0,0 \mathrm{~b}$ & $0,0 \mathrm{a}$ & $0,0 \mathrm{a}$ \\
\hline \multicolumn{5}{|c|}{ V (\%) } \\
\hline LA & $28,5 \mathrm{a}$ & 31,6 a & $43,1 \mathrm{a}$ & $61,8 b$ \\
\hline LD & $27,2 \mathrm{a}$ & $32,7 \mathrm{a}$ & $42,1 \mathrm{a}$ & $70,6 a$ \\
\hline $\mathrm{CC}$ & $25,0 \mathrm{a}$ & $29,2 \mathrm{a}$ & $42,5 \mathrm{a}$ & $59,2 \mathrm{~b}$ \\
\hline \multicolumn{5}{|c|}{ CTC $\left(\mathrm{cmol}_{\mathrm{c}} \mathrm{dm}^{-3}\right)$} \\
\hline LA & $4,54 \mathrm{a}$ & $4,44 \mathrm{a}$ & $4,46 a$ & $4,56 a$ \\
\hline LD & $4,72 \mathrm{a}$ & $4,39 a$ & $4,32 a b$ & $3,91 \mathrm{~b}$ \\
\hline $\mathrm{CC}$ & $4,36 a$ & $4,38 \mathrm{a}$ & $3,96 \mathrm{~b}$ & $4,25 a b$ \\
\hline
\end{tabular}

Tabela 3. Atributos químicos do solo determinados antes da realização do experimento

\begin{tabular}{|c|c|c|c|c|c|c|c|c|c|c|c|c|}
\hline \multirow{2}{*}{$\mathrm{pH}_{\mathrm{H} 2 \mathrm{O}}$} & $\mathbf{P}$ & K & $\mathrm{Na}$ & $\mathrm{Ca}$ & $\mathrm{Mg}$ & Al & $\mathrm{H}+\mathrm{Al}$ & SB & CTC & V & $m$ & ISNa \\
\hline & \multicolumn{3}{|c|}{$\left(\mathrm{mg} \mathrm{dm}^{-3}\right)$} & \multicolumn{6}{|c|}{$\left(\mathrm{cmol}_{\mathrm{c}} \mathrm{dm}^{-3}\right)$} & \multicolumn{3}{|c|}{$(\%)$} \\
\hline 4,7 & 1,0 & 27,0 & 4,0 & 0,2 & 0,1 & 0,6 & 4,3 & 0,38 & 4,71 & 8,1 & 11,0 & 0,4 \\
\hline
\end{tabular}


somente na dosagem de 300\% quando o resíduo LD apresentou a maior redução em virtude, provavelmente, do seu maior PRNT, além de elevados teores de $\mathrm{CaO}$ e $\mathrm{MgO}$ quando comparado ao CC e LA. Outro ponto relevante dessa informação é a acidez não trocável, correspondente ao íon hidrogênio em ligação covalente com as frações orgânicas e minerais do solo, cujo valor é obtido pela subtração do Ale do H + Al. Embora ela não seja prejudicial às plantas sua neutralização contribui para o aumento da retenção de cátions no solo (Lopez \& Guilherme, 2004) o que se verifica com a adição de todas as fontes de corretivo e dosagens utilizadas.

Observa-se aumento da CTC devido à correção da acidez não trocável correspondente a 40, 55 e 42\%, respectivamente, para LA, LD e CC na dosagem de 300\%. De acordo com Camargo et al. (1997) a elevação de um ponto na escala de $\mathrm{pH}$ eleva a CTC em 50\%; assim, todas as fontes de corretivo contribuíram para o aumento da capacidade de retenção de cátions (CTC) embora em quantitativos diferentes, sendo que LD atinge o máximo da CTC na dosagem de 75\%, diminuindo a partir dessa dosagem. Convém lembrar que os valores de CTC encontrados são muito baixos o que pode ser atribuído às argilas componentes desse solo, que são de baixa atividade (possivelmente caulinita e sesquióxidos de $\mathrm{Fe}$ e $\mathrm{Al}$ ) além do baixo teor de matéria orgânica e elevado teor de areia, que são típicos da região sul do estado do Espírito Santo (Lopez \& Guilherme, 2004).

Um fato que chama a atenção é a velocidade de reação de todas as fontes de corretivos utilizadas, evento passível de ser atribuído a dois fatores: primeiro, à homogeneidade de incorporação dos corretivos ao solo e segundo, à umidade do solo, que permaneceu sempre próxima à capacidade de campo.

\section{Influência das doses aplicadas}

$\mathrm{O}$ efeito de todos os materiais testados com relação à dose aplicada é semelhante: causa aumento linear para o $\mathrm{pH}, \mathrm{Mg}$ e V e decréscimo linear para $\mathrm{H}+\mathrm{Al}$ (Figura 1). Com relação ao pH, o ajuste obtido para o resíduo LD evidenciou maior velocidade de reação em referência aos demais corretivos. Nos teores de $\mathrm{Mg}$ a menor elevação ocorreu com o uso do CC, seguido do resíduo LA e tendo como o mais eficaz oLD. Todos os materiais testados agiram de forma semelhante no aumento da saturação de bases; no entanto, neste caso também o LD mostrou reatividade mais efetiva. $\mathrm{O}$ parâmetro $\mathrm{H}+\mathrm{Al}$ diminuiu de forma semelhante em função das doses aplicadas destacando-se também o LD como o mais eficaz. Esses resultados são concordantes com os dados de caracterização das fontes de corretivo (Tabela 2) que evidenciam o resíduo LD como o mais reativo, com maior PRNT e maior concentração de $\mathrm{Mg}$.

Para os parâmetros Ca e SB não há diferença para as fontes de corretivos utilizadas mas o modelo que melhor se adapta às variações observadas é linear decrescente para as doses utilizadas (Figura 2).

Não foi possível obter um modelo estatístico que ajustasse os resultados obtidos para o $\mathrm{Al}$, m e CTC de forma satisfatória, razão por que se optou por aplicar o teste de Tukey visando estudar o efeito das doses dentro de cada tipo de material testado (Tabela 5). A aplicação do CC reduziu o teor e a saturação de $\mathrm{Al}$ a zero na menor dose aplicada enquanto os resíduos necessitaram de dosagens maiores. O resíduo LD
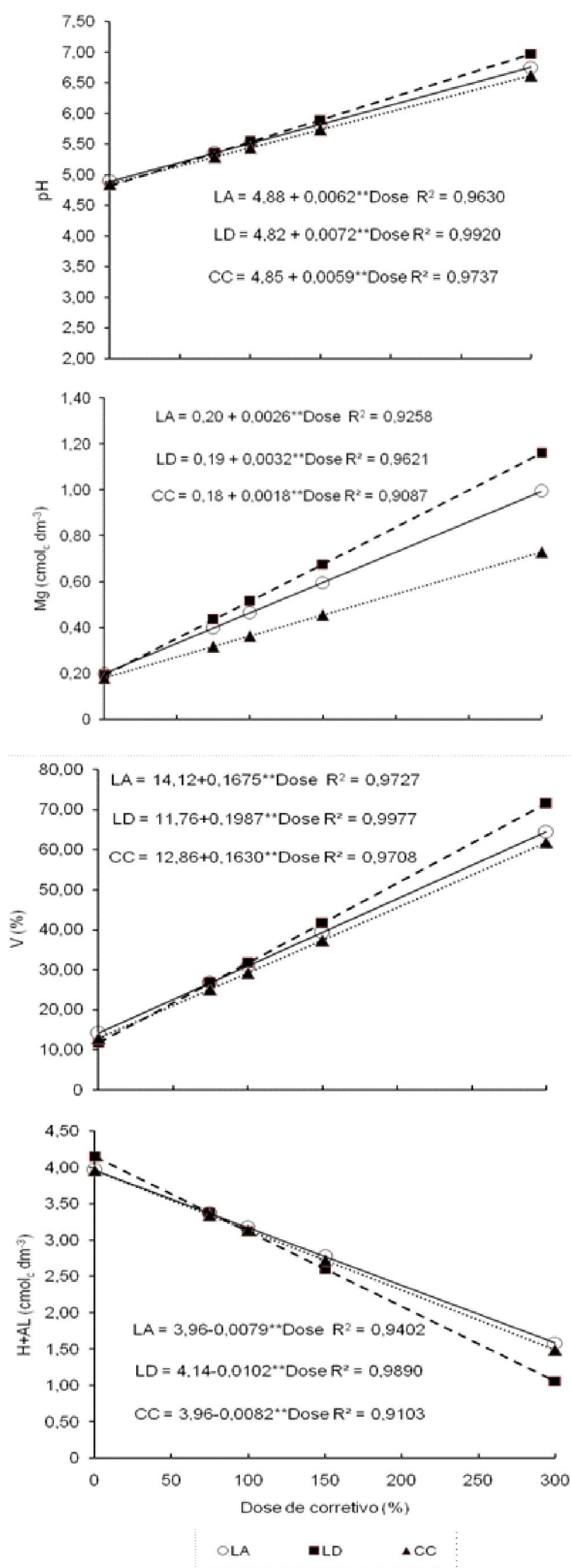

** significativo a $1 \%$ de probabilidade pelo teste $t$ de Student

Figura 1. Variação de $\mathrm{pH}, \mathrm{Mg}, \mathrm{V} \mathrm{e} \mathrm{H}+\mathrm{Al}$ em função da dose (LA = resíduo do tear de lâmina de aço; LD - resíduo do tear de lâmina diamantada; CC - calcário comercial) 


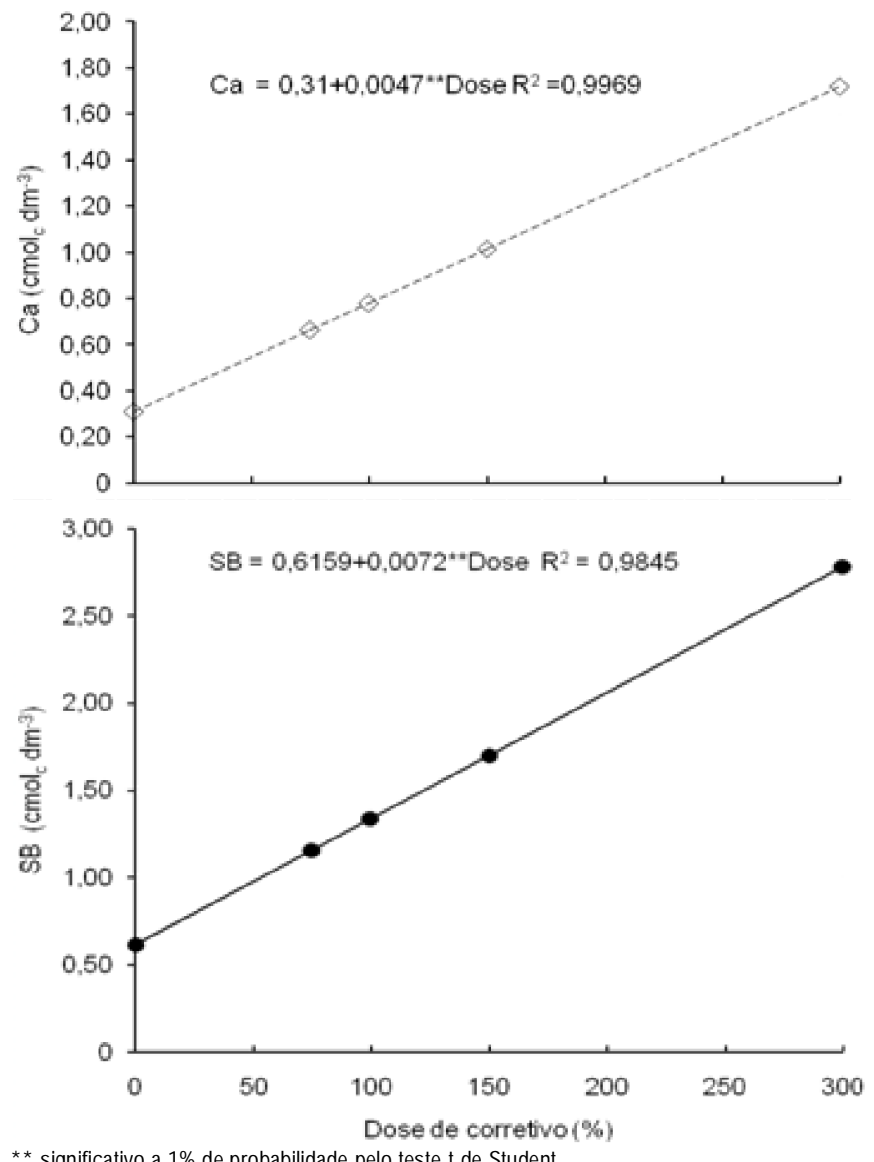

Figura 2. Variação do Ca e SB em função da dose

Tabela 5. Valores médios dos parâmetros analisados no solo tratado com resíduo do tear com lâmina de aço (LA), resíduo do tear diamantado (LD) e calcário comercial (CC)

\begin{tabular}{|c|c|c|c|}
\hline Doses (\%) & LA & LD & CC \\
\hline \multicolumn{4}{|c|}{ CTC $\left(\mathrm{cmol}_{\mathrm{c}} \mathrm{dm}^{-3}\right)$} \\
\hline $0 *$ & $4,74 a$ & 4,74 a & $4,74 \mathrm{a}$ \\
\hline 75 & $4,54 \mathrm{~b}$ & $4,72 \mathrm{a}$ & $4,36 \mathrm{~b}$ \\
\hline 100 & $4,44 \mathrm{~b}$ & $4,39 \mathrm{~b}$ & $4,38 \mathrm{~b}$ \\
\hline 150 & $4,46 \mathrm{~b}$ & $4,32 \mathrm{~b}$ & $3,96 \mathrm{c}$ \\
\hline 300 & $4,56 \mathrm{~b}$ & $3,91 \mathrm{c}$ & $4,25 \mathrm{~b}$ \\
\hline \multicolumn{4}{|c|}{$\mathrm{Al}\left(\mathrm{cmol}_{\mathrm{c}} \mathrm{dm}^{-3}\right)$} \\
\hline 0* & $0,6 a$ & $0,6 a$ & $0,6 a$ \\
\hline 75 & $0,1 \mathrm{~b}$ & $0,1 \mathrm{~b}$ & $0,0 \mathrm{~b}$ \\
\hline 100 & $0,1 b c$ & $0,0 \mathrm{C}$ & $0,0 \mathrm{~b}$ \\
\hline 150 & $0,0 \mathrm{~cd}$ & $0,0 \mathrm{c}$ & $0,0 \mathrm{~b}$ \\
\hline 300 & $0,0 \mathrm{~d}$ & $0,0 \mathrm{C}$ & $0,0 \mathrm{~b}$ \\
\hline \multicolumn{4}{|c|}{$\mathrm{m}(\%)$} \\
\hline $0 *$ & $52,5 \mathrm{a}$ & $52,5 a$ & $52,5 \mathrm{a}$ \\
\hline 75 & $8,2 \mathrm{~b}$ & $9,7 \mathrm{~b}$ & $0,0 \mathrm{~b}$ \\
\hline 100 & $3,5 \mathrm{c}$ & $0,9 c$ & $0,0 \mathrm{~b}$ \\
\hline 150 & $0,6 \mathrm{~cd}$ & $0,0 \mathrm{C}$ & $0,0 \mathrm{~b}$ \\
\hline 300 & $0,0 \mathrm{~d}$ & $0,0 \mathrm{C}$ & $0,0 \mathrm{~b}$ \\
\hline
\end{tabular}

Letras iguais na coluna não diferem significativamente entre si pelo teste de Tukey $(p<5 \%)$ * Testemunha

provocou neutralização do $\mathrm{Al}$ na dose recomendada para calagem. Com relação à CTC, houve redução dos valores em função da dose do resíduo LD; as amostras tratadas com LA e
CC não apresentaram diferenças significativas mas foi notória a redução entre a testemunha e os tratamentos. De fato, alguns trabalhos já foram conduzidos objetivando-se a correção de pH de solos acidificados em áreas mineradas em que a utilização de pó de mármore foi eficiente na redução de metais tóxicos, não apenas do $\mathrm{Al}$, mas também $\mathrm{As}, \mathrm{Cu}, \mathrm{Pb}, \mathrm{Zn}$ e Cd (FernándezCaliania \& Barba-Briosob, 2010). Em associação com compostos orgânicos esses materiais podem, inclusive, contribuir para a formação de solo em áreas completamente degradadas (Zanuzzi et al., 2009).

A Tabela 6 sintetiza os resultados considerando-se a eficiência que cada material teve na elevação ou diminuição dos parâmetros analisados na menor dose aplicada. Nota-se que o resíduo LD foi o mais eficiente na maior parte dos casos, com apenas duas situações de eficiência intermediária (para Al e m); o resíduo LA e o CC tiveram eficiência semelhante, considerando-se o número de vezes em que atuaram com a menor eficiência. É importante frisar que o fato de apresentar menor eficiência não implica em ineficiência na função de corretivo; o termo está sendo utilizado apenas com caráter comparativo. A maior eficiência do resíduo LD também se reflete aqui como resultado de sua maior reatividade e valor do PRNT, conforme dados da Tabela 2. No caso do Ca e da SB, os materiais não apresentaram diferença significativa entre si e todos foram considerados de alta eficiência na melhoria dos parâmetros, em relação à testemunha.

Tabela 6. Influência dos materiais testados na elevação ou diminuição dos parâmetros analisados

\begin{tabular}{cccc}
\hline Parâmetro & $\mathrm{CC}$ & $\mathrm{LA}$ & $\mathrm{LD}$ \\
$\mathrm{pH}$ & $\mathrm{ME}(-)$ & $\mathrm{El}$ & $\mathrm{ME}(+)$ \\
$\mathrm{Ca}$ & $\mathrm{ME}(+)$ & $\mathrm{ME}(+)$ & $\mathrm{ME}(+)$ \\
$\mathrm{Mg}$ & $\mathrm{ME}(-)$ & $\mathrm{El}$ & $\mathrm{ME}(+)$ \\
$\mathrm{Al}$ & $\mathrm{ME}(+)$ & $\mathrm{ME}(-)$ & $\mathrm{El}$ \\
$\mathrm{M}$ & $\mathrm{ME}(+)$ & $\mathrm{ME}(-)$ & $\mathrm{El}$ \\
$\mathrm{H}+\mathrm{Al}$ & $\mathrm{El}$ & $\mathrm{ME}(-)$ & $\mathrm{ME}(+)$ \\
$V$ & $\mathrm{ME}(-)$ & $\mathrm{El}$ & $\mathrm{ME}(+)$ \\
$\mathrm{SB}$ & $\mathrm{ME}(+)$ & $\mathrm{ME}(+)$ & $\mathrm{ME}(+)$ \\
\hline
\end{tabular}

CC - calcário comercial; LA - resíduo do tear de lâmina de aço; LD - resíduo do tear de lâmina diamantada; $\mathrm{ME}(+)$ - maior eficiência; EI - eficiência intermediária; $M E(-)$ - menor eficiência

\section{Situação em relação às exigências para cultivo do milho}

O solo original era pobre em nutrientes apresentando baixa CTC e deficiência de P, K, Ca e Mg devido ao alto grau de intemperismo. De acordo com a classificação da EMBRAPA (2010) o pH original era baixo $(4,5$ a 5,4) e a classificação foi mantida na aplicação da dose de $75 \%$; foi elevado para bom (5,5 a 6) nas doses de 100 e $150 \%$ e chegou a alto $(6,1$ a 7,0$)$ na dose de $300 \%$. A elevação do pH foi mais eficiente nas amostras tratadas com os resíduos LAe LD. Tal eficiência está relacionada com o maior fracionamento das partículas juntamente com teores mais elevados de $\mathrm{Ca}$ e $\mathrm{Mg}$, o que é corroborado pela caracterização desses materiais em relação à classificação de corretivos de acidez (Tabela 1). Efeito semelhante foi observado em estudos correlacionando fracionamento das partículas e variação de pH conduzidos por Prado et al. (2004), Kurihara et al. (1999) e Bellingieri et al. (1989).

O nível de Cano solo foi elevado de muito baixo (d" 0,40 $\left.\mathrm{cmol}_{\mathrm{c}} \mathrm{dm}^{-3}\right)$ para baixo $\left(0,41\right.$ a $\left.1,2 \mathrm{cmol}_{c} \mathrm{dm}^{-3}\right)$ nas doses de $75 \mathrm{a}$ 
$150 \%$, chegando ao nível médio $\left(1,21\right.$ a $\left.2,4 \mathrm{cmol}_{\mathrm{c}} \mathrm{dm}^{-3}\right)$ na dose de $300 \%$.

Todos os materiais testados elevaram o $\mathrm{Mg}$ do nível muito baixo (d" $\left.0,15 \mathrm{cmol}_{\mathrm{c}} \mathrm{dm}^{-3}\right)$ para o nível médio $\left(0,46 \mathrm{a}_{0}, 90 \mathrm{cmol}_{\mathrm{c}}\right.$ $\mathrm{dm}^{-3}$ ) nas doses de 100 e $150 \%$; na dose $300 \%$ o CC apresentou nível médio enquanto os resíduos LA e LD apresentaram resultado inverso.

A aplicação de qualquer dos materiais reduziu o teor de $\mathrm{Al}$ do nível médio $\left(0,51\right.$ a $\left.1,00 \mathrm{cmol}_{c} \mathrm{dm}^{-3}\right)$ para o nível muito baixo $\left(0,1 \mathrm{a} 0,0 \mathrm{cmol}_{\mathrm{c}} \mathrm{dm}^{-3}\right)$ na menor dose aplicada (75\%). O mesmo foi observado no comportamento de $\mathrm{m}$, em que todos os materiais testados levaram este parâmetro do nível bom $(50,1$ a $75,0 \%$ ) para o nível muito baixo $(<15 \%)$; já na dose de $75 \%$ isto indica que os resíduos possuem reatividade adequada para a neutralização do $\mathrm{Al}$ nas doses indicadas.

Todos os materiais só reduziram o $\mathrm{H}+\mathrm{Al}$ do nível médio

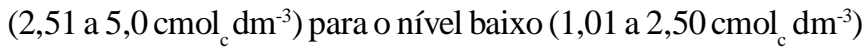
a partir da dose de $150 \%$. Diferente do comportamento da neutralização do $\mathrm{Al}$, o $\mathrm{H}+\mathrm{Al}$ não respondeu exatamente na mesma sequência da granulação dos materiais. Em pesquisa realizada por Prado et al. (2003) avaliando efeito de calcário e escória de aciariana neutralização da acidez do solo, alterações químicas significativas também foram observadas, exceto para as variáveis $\mathrm{H}+\mathrm{Al}$ e V.

Constatou-se elevação linear da $\mathrm{V}$ em todos os casos passando de muito baixa (d" 20,0\%) na testemunha, para baixa $(20,1$ a $40,0 \%)$ na dose recomendada $(100 \%)$, média $(40,1$ a $60,0 \%)$ na dose $150 \%$ e chegando ao nível bom $(60,1$ a $80,0 \%)$ na dose de $300 \%$.

O resíduo LA manteve a CTCmédia $\left(4,74 \mathrm{cmol}_{\mathrm{c}} \mathrm{dm}^{-3}\right)$ do solo original em todas as doses aplicadas. O CC provocou queda da CTC para o nível baixo $\left(4,38\right.$ a $\left.3,96 \mathrm{cmol}_{\mathrm{c}} \mathrm{dm}^{-3}\right)$ a partir da dose inicial de $75 \%$ e o resíduo LD provocou uma contínua redução da CTC alcançando o nível baixo na dose de $150 \%$ $\left(4,32 \mathrm{cmol}_{\mathrm{c}} \mathrm{dm}^{-3}\right)$.

Todos os materiais elevaram a SB do nível muito baixo (d"

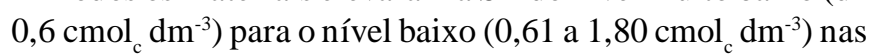
doses de 75,100 e $150 \%$ e para o nível médio $\left(1,81\right.$ a 3,60 $\mathrm{cmol}_{c}$ $\mathrm{dm}^{-3}$ ) na dose de $300 \%$.

Nos experimentos realizados por Rheinheimer et al. (2000) com cultura de milho no sistema plantio direto, a aplicação superficial de calcário aumentou o $\mathrm{pH}$, os teores de Ca e $\mathrm{Mg}$ e diminuiu os teores de $\mathrm{Al}$ trocável até a profundidade de $5 \mathrm{~cm}$ após 18 meses da sua aplicação sem, no entanto, aumentar os rendimentos do milho. Corrêa et al. (2007) observaram, utilizando resíduos (escória de aciaria, lama cal e lodo de esgoto centrifugado) para correção de $\mathrm{pH}$ de solos, efeitos positivos até $40 \mathrm{~cm}$ de profundidade com apenas três meses de reação. Para Kaminski et al. (2005) a incorporação do calcário antes da implantação do sistema plantio direto é capaz de neutralizar a acidez em profundidades maiores e sendo mais eficiente que a aplicação superficial.

Efeitos positivos da calagem na produção de milho foram observados por Caires et al. (2004) que relatam um aumento de $13 \%$ na produtividade podendo chegar a $17 \%$ quando associada ao gesso agrícola. A maior produtividade foi relacionada com o aumento da saturação por Ca nas camadas superficiais do solo.
Tais constatações podem ser modificadas positivamente com o uso do resíduo de serragem de mármore considerando-se sua maior reatividade em relação ao calcário comumente utilizado. É recomendável, portanto, que estudos similares sejam feitos para observar seus efeitos ao longo do perfil do solo.

\section{ConClusõES}

1. Os resíduos de mármore serrado com lâmina diamantada (LD) e com lâmina de aço (LA) podem ser classificados como dolomíticos apresentando características de calcário "filler" por ter granulação mais fina do que $0,30 \mathrm{~mm}$ e reatividade igual ou superior a $100 \%$.

2. Tanto a aplicação dos resíduos quanto do calcário comercial levou as concentrações de $\mathrm{Ca}$ e $\mathrm{Mg}$ e o pH do solo, eliminando a toxicidade de Al. Portanto, todos os materiais testados possuem reatividade adequada para serem utilizados como corretivo de acidez de solos.

3. O resíduo LA fornece os maiores valores de SB ao solo, e o CC, os menores valores.

4. O aproveitamento dos resíduos LA e LD mostra-se potencialmente eficaz para a prática de calagem do latossolo estudado nas doses recomendadas para cultivo de milho, observando-se as condições adotadas nesta pesquisa.

\section{Agradecimentos}

Os autores agradecem ao SINDIROCHAS e ao SEBRAE (Convênio 064/07) pelo financiamento do projeto de pesquisa que deu origem a este trabalho.

\section{LITERATURA CITADA}

Abreu Júnior, C. H.; Muraoka, T.; Lavorante, A. F. Relationship between acidity and chemical properties of Brazilian soils. Scientia Agricola, v.60, p.337-343, 2003.

Almeida, S. L. M.; Chaves, A. P. Aproveitamento de rejeitos de pedreiras de Santo Antonio de Pádua - RJ, Série Rochas e Minerais, Rio de Janeiro: CETEM/MCT, 2002. 69p.

Bellingieri, P. A.; Alcarde, J. C.; Souza, E. C. A. de. Eficiência relativa de diferentes frações granulométricas de calcários na neutralização da acidez dos solos, avaliada em laboratório. Anais da Escola Superior de Agricultura "Luiz de Queiroz", v.46, p.303-317, 1989.

Braga, F. S.; Buzzi, D. C.; Couto, M. C. L.; Lange, L. C. Caracterização ambiental de lamas de beneficiamento de rochas ornamentais. Engenharia Sanitária e Ambiental, v.15, p.237-244, 2010.

Brasil. Ministério da Agricultura, Pecuária e Abastecimento. Laboratório Nacional de Referência Vegetal. Análise de corretivos, fertilizantes e inoculantes: Métodos oficiais. Brasília: MAPA, 1983. 104p.

Caires, E. F.; Kusman, M. T.; Barth, G.; Garbuio, F. J.; Padilha, J. M. Alterações químicas do solo e resposta do milho à calagem e aplicação de gesso. Revista Brasileira de Ciência do Solo, v.28, p.125-136, 2004. 
Camargo, O. A.; Castro, O. M.; Vieira, S. R.; Quaggio, J. A. Alteração de atributos químicos do horizonte superficial de um latossolo e um podzólico com a calagem. Scientia Agrícola, v.54, p.1-8,1997.

Calmon, J. L.; Silva, S. A. C. Mármore e granito no Espírito Santo: Problemas ambientais e soluções. In: Domingues, A. F.; Boson, P. H. G.; Alípaz, S. A gestão de recursos hídricos e a mineração. Brasília: Agência Nacional de Águas - ANA, Instituto Brasileiro de Mineração - IBRAM, 2006. p.199231.

CFSEMG - Comissão de Fertilidade do Solo do Estado de Minas Gerais. Recomendações para o uso de corretivos e fertilizantes em Minas Gerais: $5^{\text {a }}$ aproximação. Viçosa: UFV, 1999.359p.

Corrêa, J. C.; Büll, L. T.; Crusciol, C. A. C.; Marcelino, R; Mauad, M. Correção da acidez e mobilidade de íons em Latossolo com aplicação superficial de escória, lama cal, lodos de esgoto e calcário. Pesquisa Agropecuária Brasileira, v.42, p.1307-1317, 2007.

EMBRAPA - Empresa Brasileira de Pesquisa Agropecuária. Manual de métodos de análise de solo. 2.ed. Rio de Janeiro: EMBRAPA, 1997. 212p.

EMBRAPA-Empresa Brasileira de Pesquisa Agropecuária. Sistemas de produção: cultivo do milho. 6.ed. Brasília: Embrapa Milhoe Sorgo, 2010. <http://sistemasdeproducao.cnptia.embrapa.br/ FontesHTML/Milho/CultivodoMilho/feranalise.htm>. 14 Abr. 2011.

Fernández-Caliania, J. C.; Barba-Briosob, C. Metal immobilization in hazardous contaminated mine soils after marble slurry waste application: A field assessment at the Tharsis mining district (Spain). Journal of Hazardous Materials, v.181, p.817-826, 2010.

Flora, L. P. D.; Ernani, P. R.; Cassol, P. C. Mobilidade de cátions e correção da acidez de um cambissolo em função da aplicação superficial de calcário combinado com sais de potássio. Revista Brasileira de Ciência do Solo, v.31, p.15911598, 2007.

Fullin, E. A.; Dadalto, G. G. Avaliação da fertilidade do solo e do estado nutricional das plantas. In: Dadalto, G.G.; Fullin, E. A. Manual de recomendação de calagem e adubação para o estado do Espírito Santo: $4^{\mathrm{a}}$ aproximação. Vitória: SEEA/ INCAPER, 2001. 26p.

Fyfe, W.; Leonardos, O. H.; Theodoro, S. C. H. Sustainable farming with native rocks: the transition without revolution. Anais da Academia Brasileira de Ciências, v.4, p.715-720, 2006.

Kaminski, J.; Rheinheimer, D. S.; Gatiboni, L. C.; Brunetto, G.; Silva, L. S. Eficiência da calagem superficial e incorporada precedendo o sistema plantio direto em um argissolo sob pastagem natural. Revista Brasileira de Ciência do Solo, v.29, p.573-580, 2005.
Kurihara, C. H.; Maeda, S.; Hernani, L. C.; Silva, W. M. Eficiência relativa de frações granulométricas de calcários sul-matogrossenses. Pesquisa Agropecuária Brasileira, v.34, p.14431449, 1999.

Lopez, A. S.; Guilherme, L. R. G. Interpretação de análise de solo: conceitos e aplicações. Boletim técnico no ${ }^{\circ}$. Associação Nacional para Difusão de Adubos. 2004. 50 p. <http://www.anda.org.br/ index.php?mpg=06.05.00\&ver=por >.09 Set. 2012.

Manhães, J. P. V. T.; Holanda, J. N. F. Caracterização e classificação de resíduo sólido "pó de rocha granítica" gerado na indústria de rochas ornamentais. Química Nova, v.31, p.1301-1304, 2008.

Moreira, J. M. S.; Freire, M. N.; Holanda, J. N. F. Utilização de resíduo de serragem de granito proveniente do estado do Espírito Santo em cerâmica vermelha. Cerâmica, v.49, p.262267, 2003.

Natale, W.; Prado, R. M.; Rozane, D. E.; Romualdo, L. M. Efeitos da calagem na fertilidade do solo e na nutrição e produtividade da goiabeira. Revista Brasileira de Ciência do Solo, v.31, p.1475-1485, 2007.

Prado, R. M.; Fernandes, F. M.; Natale, W. Efeito residual da escória de siderurgia como corretivo de acidez do solo na soqueira de cana-de-açúcar. Revista Brasileira de Ciência do Solo, v.27, p.287-296, 2003.

Prado, R. M.; Natale, W.; Fernandes, F. M.; Correa, M. C. M. Reatividade de uma escória de siderurgia em um latossolo vermelho distrófico. Revista Brasileira de Ciência do Solo, v.28, p.197-205, 2004.

Ramos, L. A.; Nolla, A.; Korndörfer, G. H.; Pereira, H. S.; Camargo, M. S. Reatividade de corretivos da acidez e condicionadores de solo em colunas de lixiviação. Revista Brasileira de Ciência do Solo, v.30, p.849-857, 2006.

Rheinheimer, D. S.; Santos, E. J. S.; Kaminski, J.; Xavier, F. M. Aplicação Superficial de calcário no sistema plantio direto consolidado em solo arenoso. Ciência Rural, v.30, p.263268, 2000.

Silva, E. A. da; Cassiolato, A. M. R.; Maltoni, K. L.; Scabora, M. H. Efeitos da rochagem e de resíduos orgânicos sobre aspectos químicos e microbiológicos de um subsolo exposto e sobre o crescimento de Astronium fraxinifolium Schott. Revista Árvore, v.32, p.323-333, 2008.

Straaten, P. V. Farming with rocks and minerals: Challenges and opportunities. Anais da Academia Brasileira de Ciências, v.78, p.731-747, 2006.

Theodoro, S. H.; Leonardos, O. H.The use of rocks to improve family agriculture in Brazil. Anais da Academia Brasileira de Ciências, v.78, p.721-730, 2006.

Zanuzzi, A.; Arocena, J.M.; Van Mourik, J.M.; Faz Cano, A. Amendments with organicand industrial wastes stimulate soil formation in mine tailings as revealed by micromorphology. Geoderma, v.154, p.69-75, 2009. 